\title{
LOS CONCEPTOS DE MUERTE Y MORIR EN ENFERMERÍA $Y$ LAS RELACIONES CON EL ETHOS DEL CUIDADO
}

\section{OS CONCEITOS DE MORTE E MORRER NA ENFERMAGEM E AS RELACÓES COM O ETHOS DO CUIDADO}

\author{
*Lopes Magalháes, AV. **Lyra da Silva, RC. \\ *Enfermera. **Profesor Asistente Departamento de Enfermagem Fundamental da Escola de \\ Enfermagem Alfredo Pinto- UNIRIO. Brasil.
}

Palabras clave: Cuidado de Enfermería, Muerte, Sector de asistencia a la salud

Palavras chave: Cuidado de Enfermagem, Morte, Setor de Assisténcia a Saúde.

\section{RESUMEN}

En este estudio analizamos la influencia de los conceptos de muerte y morir del equipo de Enfermería sobre las prácticas de cuidados en algunos espacios de internación hospitalaria. Los datos se produjeron a partir de la aplicación de un formulario conteniendo preguntas abiertas, siendo los sujetos de la investigación Enfermeros, Técnicos y Auxiliares de Enfermería que actúan en los sectores de Clínica Médica, Unidad de Terapia Intensiva y Emergencia de hospitales de la red pública localizados en el municipio de Rio de Janeiro. Después del análisis de los datos, emergieron dos categorías: la INFLUENCIA y el CONFLICTO de los conceptos acerca de la muerte en la ELECCIÓN y en la DINÁMICA de del ethos del cuidado, y Muerte: INICIO de una nueva vida y FIN de todo. Los resultados apuntan que los conceptos de muerte no se alteran entre los profesionales de enfermería que actúan en esas unidades investigadas y también parece no haberles influenciado cuando eligieron sus ethos para cuidar. Llama la atención que los profesionales entrevistados relataron conflicto en el pensar la muerte y en encararla en su cotidianidad de cuidar.

\section{RESUMO}

Neste estudo, analisamos a influencia dos conceitos de morte e morrer da equipe de Enfermagem sobre as práticas de cuidar em alguns espacos de internacáo hospitalar. Os dados foram produzidos a partir da aplicacáo de um formulario contendo perguntas abertas, sendo os sujeitos da pesquisa, Enfermeiros, Técnicos e Auxiliares de Enfermagem que atuam nos setores de Clínica Médica, Unidade de Terapia Intensiva e Emergencia de hospitais da rede pública localizados no município do Rio de Janeiro. Após a análise dos dados, emergiram duas categorias: A INFLUENCIA e o CONFLITO dos conceitos acerca da morte na ESCOLHA e na DINAMICA do ethos do cuidado; e Morte: INICIO de uma nova vida e FIM de tudo. Os resultados apontam que os conceitos de morte náo se alteram entre os profissionais de enfermagem que atuam nessas unidades pesquisadas e também parece náo ter os influenciado quando escolheram os seus ethos 
para cuidar. Chama atencáo que os profissionais entrevistados relataram confuto no pensar a morte, e de encará-la em seu cotidiano de cuidar.

\begin{abstract}
In this study, we analyze the influence of the concepts of death and dying of the nursing staff on the practice of care given in some areas of hospitalization. The data was produced by from a questionnaire containing open ended questions. The subjects of the investigation nurses, technicians and nurses' auxiliaries who act in the sectors of the medical clinic, the center of intensive care and emergency in public hospitals in the city of Rio de Janeiro, Brazil. After the analysis of the data, two categories emerged: the INFLUENCE and CONFLICT of the concepts about death in the CHOICE and DYNAMICS of ethos of care and death: BEGINNING a new life and END of everything. The results point that the concepts of do not change among the nursing professionals who work in the units studied, and also it seems not to have influenced them when they chose their ethos of care giving. It draws attention that professionals in these units relate a conflict when thinking of death and facing it in their daily care.
\end{abstract}

\title{
CONSIDERACIONES INICIALES
}

El cuidado, en la concepción de varios teóricos y filósofos de la actualidad, se entiende como fundamental en el mantenimiento de la vida en la tierra. La palabra cuidado se origina del verbo cogitare-cogitatus que significa curar, imaginar, pensar, dar atención, revelar una actitud de preocupación. Se puede comprender el cuidado como parte integrante de la condición humana, lo que nos lleva a creer que, sin él, difícilmente sería posible nuestra existencia. En este sentido, el término cuidado va más allá de su antónimo (descuido), transciende el interés por las cosas materiales, yendo al encuentro del espíritu o de la propia alma, que como la materia, también carece de cuidados(1).

Así, podemos entender que durante toda la existencia en la tierra, y hasta el momento de finitud, como normalmente nos referimos al momento de la muerte, carecemos de cuidados con la materia, pero, en nuestra opinión, principalmente con aquella porción que parece animarla, el alma o espíritu. Podemos entonces afirmar que el cuidado se torna indispensable al ser humano, independiente de la fase del ciclo de la vida en que se encuentre. Esté naciendo o muriendo, el cuidado deberá ser indispensable.

En estudio reciente acerca de los conceptos de muerte y morir y sus relaciones con el ethos del cuidado, se observó que el comportamiento de los profesionales de enfermería ante la muerte y el morir, cada día más, se fundamenta en principios Cartesianopositivistas, lo que influye sobre las prácticas de cuidar en enfermería, particularmente en situaciones límites, de muerte inminente(2). En este estudio, utilizamos la palabra ethos que, en su sentido originario griego, significa "la cueva del animal o casa humana" en nuestro contexto de investigación la palabra ethos, referente al cuidado, fue utilizada como "lugar', el espacio que reservamos para cuidar a alguien, en este caso el sector de actuación del profesional de enfermería en el ámbito hospitalario.

En este sentido, creemos que los conceptos previamente concebidos acerca de la muerte y del morir, y la manera por la que los profesionales de salud se relacionan con estos fenómenos, basados en sus conceptos previos, parecen determinar la forma con que ellos se relacionan con la vida, determinando entre otras cosas, la manera con que cuidan a sus clientes. 
Durante la realización de un estudio Socio-poético acerca del imaginario de los enfermeros cuando cuidan en la frontera vida/muerte (2), se encontró fuerte indicativo de que la forma con que los enfermeros imaginan y representan la muerte y el morir influye en la elección por el ethos (espacios) del cuidado. Destacamos como objeto de este estudio, los conceptos de muerte y morir en la enfermería en los diferentes espacios del cuidado que determinan la manera por la cual sus clientes son cuidados.

Así, entendemos que la comprensión de los conceptos de muerte y morir que los profesionales de enfermería adoptan cuando cuidan a sus clientes, podrá determinar la manera con que estos se relacionen con la muerte, lo que posibilitaría explicar por qué razones el cuidado de enfermería que nos parece tener la misma esencia, presenta diversas facetas, dependiendo del cliente y del espacio en que es desarrollado.

Y, presentamos como objetivos, definir los conceptos de muerte y morir de los que los profesionales de enfermería se han apropiado cuando cuidan a clientes en diferentes sectores de internación hospitalaria y analizar las posibles implicaciones que tales conceptos traen para la práctica de cuidar.

Por lo tanto, este estudio se justifica por la necesidad de una mayor profundización en las discusiones respecto al significado de los conceptos de muerte y morir entre los miembros del equipo de enfermería, en diferentes ethos del cuidado en el ambiente hospitalario. Creemos que sólo desvelando tales conceptos y significados será posible que entendamos los diferentes comportamientos de los profesionales de enfermería ante la muerte y el morir, y cuál es la relación que estos conceptos tienen con la elección del espacio en que estos profesionales irán a cuidar a sus clientes.

\section{METODOLOGÍA}

El estudio es de tipo descriptivo y se ha delineado a partir de un abordaje cualitativo. Considerando el carácter subjetivo del objeto a ser investigado, ya que los aspectos que involucran la muerte y el morir, bien como el imaginario y los valores de cada individuo, sujetos de la investigación, son básicamente determinados por los paradigmas en que cada uno, individualmente se basa(3).

"La investigación cualitativa se ocupa, en las ciencias sociales, con un nivel de realidad que no puede ser cuantificado, es decir, trabaja con el universo de significados, motivos, aspiraciones, creencias, valores y actitudes, lo que corresponde a un espacio más hondo de las reacciones, de los procesos y de los fenómenos que no pueden ser realizados en la operacionalización de las variables" (4). (MINAYO 1995:21)

Los escenarios elegidos fueron las Unidades de Terapia Intensiva (UTI), general o especializada, Emergencia (SPA) y Clínica Médica (CM) de cinco hospitales de la red pública, ubicados en la ciudad de Río de Janeiro, teniendo como sujetos de la investigación Enfermeros, Técnicos y Auxiliares.

Como estrategia de recopilación de datos, elaboramos un instrumento del tipo formulario estructurado, conteniendo preguntas abiertas. El cuantitativo de la muestra utilizada es de treinta, divididos en Enfermeros, Técnicos y Auxiliares de enfermería. 
El referencial teórico para analizar y categorizar las informaciones de los sujetos del estudio sobre sus conceptos de muerte y morir en la enfermería y la relación de estos con el ethos del cuidado fue la Socio-poética. La elección de la Socio-poética como referencial teórico ocurrió porque creemos que esa nueva ética comprende mejor, al discutirse un tema tan subjetivo y cargado de arquetipos como la muerte y el morir, ofreciendo un entendimiento más amplio de esos conceptos en lo que se refiere a la materia y al espíritu en esa gran travesía de la muerte, y sirviéndose de la multireferencialidad para discutir sus múltiples facetas, lo que no seria posible conseguir, ciertamente, con métodos racionales de abordajes teóricos ${ }^{(4)}$.

A partir del pensamiento cualitativo y multireferencial, es posible elucidar y objetivar mejor la complejidad de los hechos, de las situaciones y de las prácticas formadoras. "Problemas complejos como la muerte y el morir, por ejemplo, exigen instrumentos, campos y teóricos diversos, esto porque las situaciones son complejas por naturaleza, pues involucran un juego de imagen e imaginario" ${ }^{(5)}$.

Elucidar tales situaciones nos exige la articulación, siempre particular, interdisciplinar y multireferencial. De esta forma, el abordaje complejo de la Socio-poética viene de cara a nuestras necesidades de la investigación, por permitir desmembramientos para las varias dimensiones de la vida personal y social del ser humano, relacionados con los conceptos de muerte y morir por los sujetos estudiados en este estudio.

Vale resaltar que se solicitó la autorización por escrito de las Instituciones y de los sujetos entrevistados que quedaron informados del objeto, objetivos, abordaje metodológico y relevancia del estudio, así como la utilización del contenido de las entrevistas y de los resultados de la misma para poderlas publicar.

\section{PRESENTACIÓN Y ANÁLISIS DE LOS DATOS}

\section{Caracterización de los Sujetos}

Elegimos demostrar, a través de gráficos, las caracterizaciones relevantes de los sujetos, para el referido estudio.

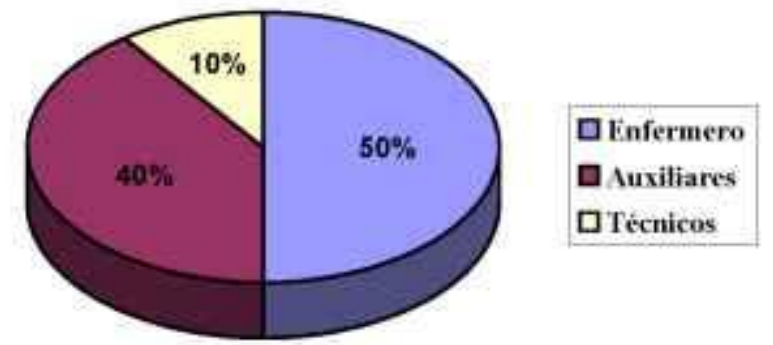

\section{Gráfico I - Distribución de los sujetos por categoria profesional}




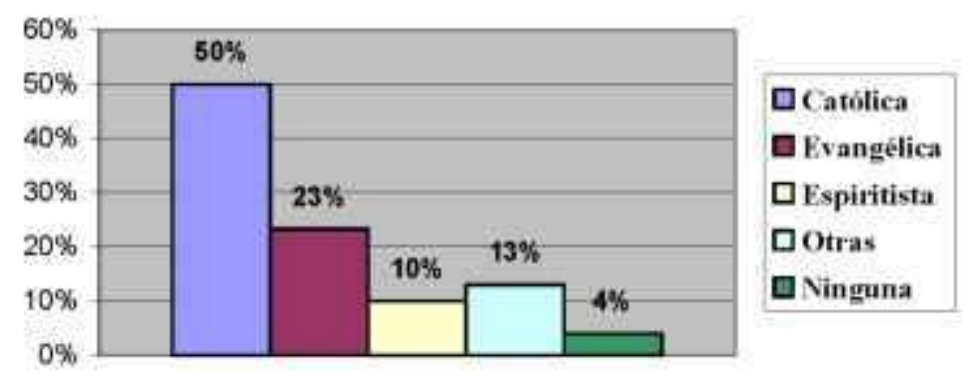

\section{Gráfico II - Distribución de las religiones declaradas}

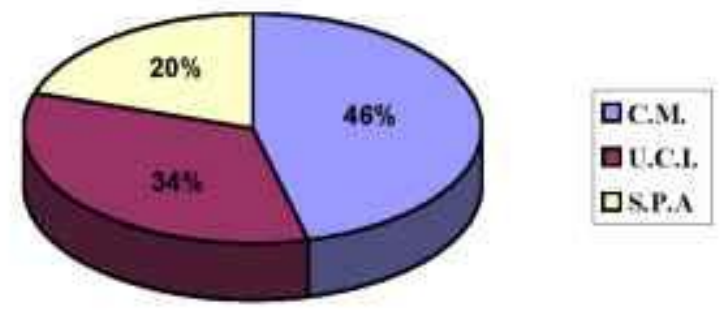

\section{Gráfico III - Distribución de los sujetos por sectores de actuación}

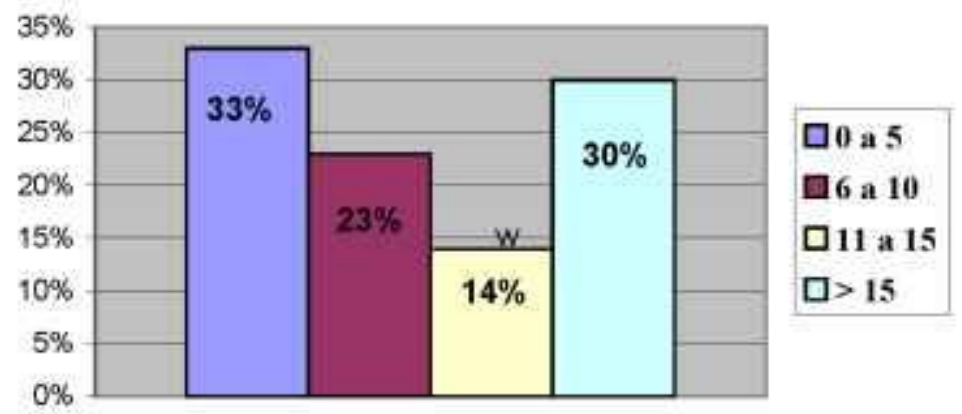

\section{Gráfico IV - Distribución de los sujetos por tiempo/año de experiencia profesional}

\section{Discusión de los datos encontrados}

Tras el análisis de los datos, emergieron dos categorías: LA INFLUENCIA y EL CONFLICTO de los conceptos de muerte y morir en la elección y dinámica en el ethos del cuidado; y Muerte: Inicio de una nueva vida y Fin de todo.

En nuestro instrumento de recolección de datos, utilizamos dos preguntas relevantes, que nos ayudaron en el análisis y en la construcción de esta categoría: la primera se refiere a la influencia de los conceptos de muerte y morir en la elección de la unidad de actuación profesional (Gráfico V); la segunda busca saber si las concepciones de muerte y morir generan conflicto con la dinámica de sus actividades en el momento de cuidar del cliente en el ambiente hospitalario (Gráfico VI).

De este modo, observamos los siguientes resultados: 


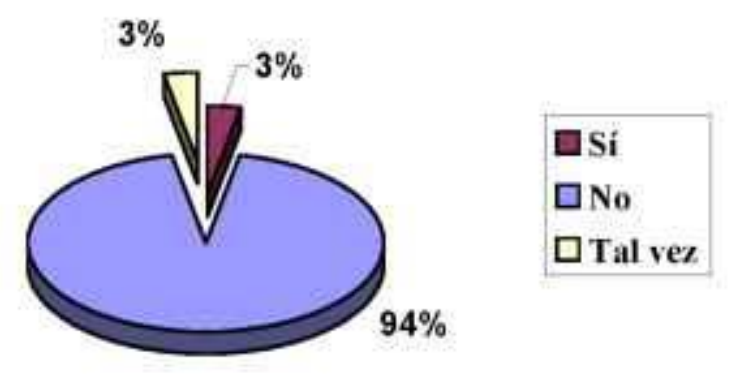

Gráfico V - Influencia del concepto de muerte y morir en la elección del ethos del cuidado.

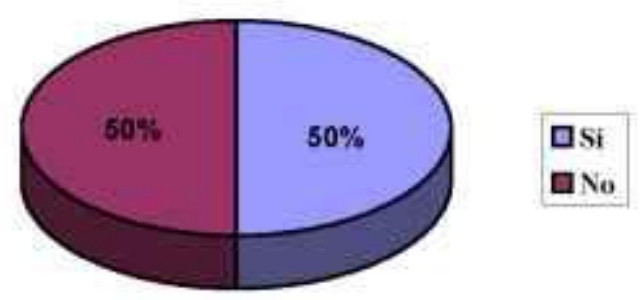

\section{Gráfico VI - Conflicto entre el concepto de muerte y morir y la dinámica del ethos del cuidado}

Podemos observar que en el $94 \%$ de los profesionales entrevistados, los conceptos que éstos tienen sobre muerte y morir no influyeron en la elección del área de actuación profesional; en el $3 \%$ de los sujetos investigados hubo la influencia del concepto de muerte en el momento de la elección del sector de actuación y los otros $3 \%$ no supieron contestar. Sin embargo, en el momento de cuidar de sus clientes, el $50 \%$ de estos profesionales relataron que tales concepciones sobre estos fenómenos crean conflicto con la dinámica de trabajo, lo que demuestra que el concepto formado, por cada profesional investigado, sobre el asunto muerte se contradice en el imaginario de estos sujetos en el momento de prestar cuidado al cliente. Por lo tanto, "la situación de trabajo suscita sentimientos muy fuertes y contradictorios" (6).

Entendemos que aunque tratan con el fenómeno de la muerte y con el proceso de morir en la práctica profesional cotidiana en las unidades de trabajo, todavía existe una dificultad, relatada por los entrevistados, para tratar con los conceptos de muerte y morir en sus imaginarios en el momento de cuidar, principalmente cuando involucra valores culturales y creencias religiosas.

Para sostener esta discusión, consideramos que:

"Teniendo en cuenta la diversidad de expresiones religiosas en nuestras culturas y la diversidad de posturas adoptadas por los enfermeros como reflejos del medio en que viven, las cuestiones relativas a los valores de los clientes y de sus familiares muchas veces perjudican la relación y la interacción entre ellos" (7). 
Con respecto a la vivencia de los profesionales de enfermería en su trabajo cotidiano con diversos tipos de personas que poseen diferentes culturas y religiones, en la convivencia con otros profesionales de la misma área de actuación o no, con los clientes y con los que están junto a ellos, como los familiares, amigos y/o acompañantes en las unidades de trabajo, principalmente en el momento del cuidado, en que debemos transmitir al cliente seguridad, comodidad y afecto, además de aplicar los conocimientos técnico-científicos, aprendimos que tratar con personas de valores culturales diferentes, conviviendo todos los días en nuestro ambiente de trabajo, nos permite ir más allá de nuestras concepciones, contradiciendo nuestros propios conceptos sobre el fenómeno de la muerte en el momento de prestar asistencia al cliente.

Considerando que además de profesionales de salud, somos seres sociales y tenemos nuestros valores culturales y religiosos, así como los clientes, familiares y acompañantes que se encuentran en las unidades de internación, vale resaltar que las creencias religiosas y los valores culturales de cada uno se refieren al sentido de la vida y la muerte y estos deben ser respetados. Sabemos que las religiones tuvieron y aún presentan gran función en la explicación de los misterios que circundan esos fenómenos a través de la fe y la creencia.

Aunque, la religión no sea nuestro enfoque principal en el estudio, no podemos dejar de lado su influencia a lo largo de toda nuestra historia. Así, confirmamos nuestra posición cuando se resalta que: "Se sabe que las convicciones religiosas son posicionamientos resistentes que despiertan verdaderas manifestaciones de pasión en sus seguidores, principalmente en lo que se trata del contexto de asistencia hospitalaria" ${ }^{(6)}$.

Sin tomar en consideración los conceptos de los profesionales acerca de muerte y morir, sabemos que en algunos sectores, la muerte en los clientes se encuentra más inminente, visible en el ámbito hospitalario. Podemos observar la muerte, comúnmente, en la "UCl.". "En la Edad Media, la muerte estaba en las salas de visitas, hoy, ella se esconde en los hospitales, en las UCIs." (i). En la unidad de cuidados intensivos encontramos clientes que necesitan más cuidado y mayor tecnología disponible, pues en ese espacio hay constantes sofisticaciones de los métodos de soporte avanzado de vida, que a veces ayudan y permiten la recuperación de innumerables personas que se encuentran próximas a la muerte; en gran número, inconcientes o con gran riesgo de vida.

Teniendo presente, la discusión de la incorporación de alta tecnología actualmente en el ámbito hospitalario, consideramos que:

"Nuevas tecnologías en el ámbito del moderno hospital a partir de la identificación de aportes tecnológicos introducidos para el perfeccionamiento de diagnósticos y terapéuticas pueden señalar algunos de los cambios significativos que el hospital viene incorporando en las últimas décadas." (6)

Para la autora, la incorporación de nuevas tecnologías en el ámbito hospitalario, acentúa el dinamismo tecnológico y asistencial esencialmente del trabajo intensivo. Sin embargo, en nuestra investigación, el concepto de muerte del profesional que actúa en $\mathrm{UCl}$, no los diferenció de los profesionales que trabajan en sectores de Clínica Médica y emergencia, quienes relatan sus conceptos de muerte y morir en el momento de prestar asistencia al cliente como: "muerte como el fin de todo, hecho inmediato que no hay cómo recuperar"; y aún: "muerte inicio de una vida para otro mundo, una nueva dimensión". Ante lo discutido, llamamos la atención de ¿por qué los conceptos de muerte y morir en los profesionales de 
enfermería, sujetos de esta investigación, no influyeron en el momento de la elección del sector trabajo?

En la segunda categoría, entendemos la muerte de dos formas. La primera, los profesionales la entienden como la separación de la materia y del espíritu. El nacimiento es una trascendencia a una nueva vida, donde es descrita por los sujetos investigadores como: "un nuevo recomienzo en otra dimensión" y "principio de una vida".

En la creencia de una continuidad de la vida tras muerte, relatada por los profesionales, observamos la importancia de no cuidar solamente del cuerpo en sí, sino algo más allá, que Ilamamos del plano espiritual.

Para BOFF (1999:151: "Cuidar del espíritu significa cuidar los valores que dan rumbo a nuestra vida y también las significaciones que generan esperanza para más allá de la muerte". $\quad$ Para el autor, el ser humano se sentirá en su plenitud con el cultivo del espacio espiritual, orientando el destino de su vida para evitar equívocos en su camino. Así, encontrará el sentido de la muerte sin afectar su vida espiritual.

Encontramos en este análisis sobre la muerte la inserción del carácter religioso y cultural de los profesionales de enfermería, los cuales no creen que el espíritu permanecerá para dar continuidad a la vida, sólo en otra dimensión, plano existencial o posteriormente un renacimiento. La creencia en otra vida tras la muerte "ameniza" el conflicto de la concepción de muerte en el momento del cuidar, pues el sentimiento de "pérdida" del cliente cuando se acerca a la muerte se sustituye por el alivio espiritual de saber que él va más allá a una nueva dimensión para el inicio de un nuevo ciclo vital.

En un estudio socio- antropológico de la muerte, Morin (8) percibió entre otras cosas que en las culturas estudiadas, la idea de muerte-renacimiento se encuentra fuertemente presente, cuando dice: toda muerte provoca un nacimiento y lo inverso; todo nacimiento provoca una muerte. Resalta aún que "por medio de las concepciones de muerte y vida, el hombre descubre al mismo tiempo su muerte y su inmortalidad".

De este modo, podemos observar que independientemente de la cultura estudiada, todos de una manera o de otra, creen en una posibilidad de vida tras la muerte, intentando ver en ella la posibilidad de inmortalidad, buscando así una forma de quedarse íntimamente conectado al mundo, sólo que en otra dimensión.

Al contrario, la segunda forma de pensar la muerte se entiende como el fin inexorable del recorrido de la vida, vista como un aniquilamiento. Algunos sujetos conceptúan como:"hecho inmediato, que no hay cómo recuperarlo", o "el final de la vida para el mundo es el fin de todo". Ésta no es formalmente sentida por esos profesionales, exactamente porque ya está en su cotidianidad de trabajo.

"El hecho de que los enfermeros asumen la muerte como algo que se desea parece más un mecanismo de defensa o una formación reactiva frente a la amarga realidad de su día a día, del ejercicio de la práctica, aun como una manera de manifestar cierta intimidad con el proceso de muerte" (1).

Entendemos que en ambientes donde la experiencia de la proximidad de la muerte es rutinaria, encarada por los profesionales como un hecho "común", entonces percibimos que al hablar respecto a este fenómeno, para algunos el camino de la muerte se separa de la 
vida y no hay una unión, siendo un proceso natural del ser humano, donde este nace, se desarrolla y después muere.

\section{CONSIDERACIONES FINALES}

Los resultados apuntan a que los conceptos de morir no se alteran entre los profesionales de enfermería que actúan en unidades de cuidados intensivos, Emergencia y Clínica médica. De este modo, encontramos en esas unidades las mismas concepciones acerca de la muerte. El concepto que la define como una realidad cierta e inexorable, determinando el fin de todo, se reafirma en el discurso de estos profesionales; sin embargo, para otros, muerte y morir se entienden como la conexión a la idea de nacimiento oriunda de su cultura en la convicción de la existencia y continuidad de la vida tras muerte.

El concepto de muerte nos pareció que no influyó en estos profesionales en la elección de sus ethos del cuidado, pero tales conceptos determinan la manera por la cual sus clientes son cuidados en estas unidades de internación hospitalaria investigadas. Llamamos la atención de que los profesionales entrevistados, los cuales militan en estas unidades, relataron el conflicto en el pensar la muerte y en encararla en su cotidianidad de cuidar, observando la diversidad de posturas adoptadas por los mismos, como reflejos del medio en el cual viven; su propio cotidianidad social, dificultando así el momento de la práctica del cuidar en el sector de actuación. Resaltamos que los aspectos religiosos fuertemente adoptados por esos sujetos influyeron en la concepción del fenómeno de la muerte.

Con eso, tras la realización de una literatura profundizada acerca de la muerte y el proceso del morir además la influencia de este fenómeno presentando contradicciones en la práctica del cuidar, debido a diversas posturas, culturas y religiones de los profesionales de enfermería y los demás seres sociales que lo cercan en su cotidianidad del trabajo, vimos la necesidad de reflexión de los valores individuales y colectivos de los mismos en lo que corresponde a la subjetividad de este fenómeno muerte y la dificultad de lucharar con el mismo que encontramos comúnmente, inserto en nuestro ambiente de trabajo, principalmente en los sectores de internación hospitalaria involucrados en este estudio.

\section{REFERENCIAS:}

1- BOFF, Leonardo. El Saber cuidar: ética del humano - compasión por la tierra. Petrópolis, RJ: Vozes, 1999.

2- SILVA, Roberto C. L. da. El imaginario de enfermeros cuando cuidan en la frontera_vida/muerte: un estudio Socio-poético, 2001. Disertación (Máster en Enfermería) - Universidad Federal del Estado de Río de Janeiro, Río de Janeiro, 2001.

3- MINAYO, Maria C. et al. Investigación social: teorías, método y creatividad. Río de Janeiro: Vozes, 1997.

4- SANTOS, I; GAUTHIER, J. Enfermagem: Análise Institucional e Sócio-Poética. Rio de Janeiro. Editora Anna Nery, 1999.

5- BORBA, Sergio. Multireferencialidad en la formación del profesor-investigador: conformidad y complejidad. Maceió: PSE, 1997. 
6- PITTA, Ana. Hospital dolor y muerte como oficio. Säo Pauio: Hucitec, 1994.

7- MACHADO, Wiiiam César Aives, LEITE, Josete Luzia. Eros e Thanatos: La Muerte sob ia Óptica de ia Enfermaría. Río de Janeiro: Ed. Difusäo, 2004.

8- MORIN, Edgar. El hombre y la muerte. Río de Janeiro: Imago, 1997. 